\title{
Adaptación y validación de escalas de medición en el trabajo. Parte 1: bienestar social
}

\author{
María L. Campos-Carreño, Camila B. Velasco y Javiera P. Araya \\ Facultad de Humanidades, Departamento de Psicología, Universidad de La Serena, La Serena-Chile. \\ (correo-e: mcampos@userena.cl; cvelasco@userena.cl; javiera.arayac@userena.cl)
}

Recibido Abr. 1, 2020; Aceptado May. 27, 2020; Versión final Jul. 27, 2020, Publicado Oct. 2020

\section{Resumen}

El objetivo de este estudio fue adaptar y validar al contexto laboral la escala de bienestar social de Keyes, para lo cual se ejecutó una investigación cuantitativa con una muestra de 332 trabajadores divididos aleatoriamente en dos submuestras (A y B). Se realizó un análisis factorial exploratorio por medio de mínimos cuadrados generalizados (submuestra A) y un análisis factorial confirmatorio con el método de estimación de mínimos cuadrados ponderados multivariantes (submuestra B). Los resultados muestran una escala de bienestar social en el trabajo con indicadores de ajuste adecuados: $\mathrm{CFI}=0.984, \mathrm{TLI}=0.98$ y $\mathrm{RMSEA}=0.091$. La escala está constituida por 3 factores: pertenencia social, interacción social y comprensión social en el trabajo, cuyos respectivos valores de omega certifican consistencia interna. Se concluye que la escala de bienestar social en el trabajo presenta características psicométricas adecuadas para su uso, constituyéndose en un aporte para la psicología del trabajo y las organizaciones.

Palabras clave: bienestar social; trabajo; escala Likert; psicología; organizaciones

\section{Measurement scale adaptation and validation in the work context. Part 1: social well being}

\begin{abstract}
The objective of the present study was to adapt and validate the Keyes social well being scale in the work context. This is a quantitative research study with a sample of 332 workers randomly assigned into two subsamples (A and $B$ ). An exploratory factorial analysis was performed using generalized least squares (subsample A). A confirmatory factorial analysis was performed with the multivariate weighted least squares estimation method (subsample B). The results showed a social well being scale in the work environment with adequate adjustment indicators: CFI (0.984), TLI (0.98), and RMSEA (0.091). The scale consists of three factors: 1) social belonging, 2) social interaction, and 3) social understanding at work. Their omega values certify internal consistency. It is concluded that the social welfare scale in the work context presents psychometric characteristics suitable for its use, making it a contribution to work and organizational psychology.
\end{abstract}

Keywords: social well-being; work; Likert scale; organizational psychology 


\section{INTRODUCCIÓN}

En el contexto internacional, distintos gobiernos están enfrentando que a pesar del aumento del Producto Interno Bruto (PIB), los niveles de satisfacción y bienestar en la población siguen estables o bajan, surgiendo así la necesidad de alejarse de un concepto puramente económico como único criterio a partir del cual medir el bienestar de los ciudadanos. Por ello la utilización de nuevas herramientas, como las encuestas de felicidad, constituyen una poderosa manera de entender el bienestar de las personas, dando así la posibilidad de direccionar de manera adecuada la política organizacional y gubernamental. (Basaure, 2013). La Organización de las Naciones Unidas (ONU) y la Organización para la Cooperación y el Desarrollo Económico (OCDE), se encuentran implementando mediciones globales de satisfacción y felicidad, cuyo objetivo es alentar a los gobiernos a incorporar dichas mediciones como una cuestión de Estado (Basaure, 2013). En el contexto nacional, el gobierno chileno ha demostrado estar en conocimiento de los desarrollos alcanzados en estas materias incorporando mediciones de felicidad en la Encuesta de Caracterización Socioeconómica Nacional (CASEN) del año 2011. Encuesta que mide tanto el bienestar objetivo (cuánto gana la persona, si tiene acceso a los servicios sociales, etc.) como el bienestar subjetivo a nivel individual y social, (si es feliz con su vida y con los sistemas sociales de pertenencia) (Basaure, 2013).

El Programa de las Naciones Unidas para el Desarrollo (PNUD) del año 2012, evaluó en Chile el Bienestar Subjetivo, constructo que definió como la valoración de las oportunidades y soportes que el entorno ofrece para desarrollar y potenciar una autoimagen satisfactoria. Los resultados revelaron alto bienestar subjetivo a nivel personal, pero bajo nivel de bienestar subjetivo a nivel social, declarando disconformidad con la sociedad en general y con las organizaciones representativas de pertenencia como el sistema político, salud, educación, etc. Malestar subjetivo social que actualmente se manifiesta de manera significativa a través de movimientos sociales masivos a lo largo del país. Como evidencia de lo señalado referimos la marcha realizada el 25 de octubre del 2019 calificada como histórica dado a que reunió sobre el millón de personas en la capital del país.

Nuestro país presenta un crecimiento económico del 120\% los últimos 15 años, una caída de la tasa de desempleo del 7\%, el primer lugar en América Latina y El Caribe en desarrollo humano, y un aumento significativo en los niveles de inversión y emprendimiento, indicadores que se contraponen a los altos índices de malestar social. Este malestar se acompaña por la ubicación de nuestro país entre las naciones más enfermas mentalmente del planeta, a lo cual se suma según la OCDE el segundo lugar a nivel mundial en el aumento de tasa de suicidios, y altos niveles de depresión y ansiedad (Martínez y Zamora 2013).

Los estudios sobre bienestar se han basado en tres aspectos: la satisfacción con la vida; el bienestar hedónico, el que hace referencia al placer, disfrute, confort, estrés, a la satisfacción y reacción afectivas de los sujetos; y el bienestar eudaimónica que se centra en el crecimiento, desarrollo personal orientado al logro de complejas metas vitales, las cuales poseen un alto grado de significado personal (Ryff y Keyes, 1995; Arévalo-Avecillas, Game, Padilla-Lozano y Wong, 2019; Czerw, 2019). La relevancia del constructo en estudio ha generado que diversos investigadores levanten validaciones de Instrumentos de Bienestar. Sin embargo, hasta ahora los esfuerzos científicos se han focalizado en la perspectiva hedónica y enmarcados fuera del contexto laboral (Czrew, 2019). Es por eso que el presente estudio se inserta en la conceptualización eudaimónica del bienestar y focalizado en el contexto laboral.

\section{OTROS ANTECEDENTES}

La comprensión del bienestar implica lo individual y lo social a partir de lo experimentado por un sujeto sociohistórico que vive en una sociedad la cual brinda satisfacción a las necesidades y aspiraciones que este posee (Ivankina y Latygovskaya, 2015). A su vez, implica la existencia de un cierto estándar que corresponde al sistema de valores aceptados por una cultura en una determinada época (Ivankina y Latygovskaya, 2015). Keyes (1998) define el bienestar social como la "valoración que los individuos hacen de las circunstancias y el funcionamiento dentro de la sociedad" y sostiene que aquellas personas que poseen un mayor bienestar social se caracterizan por: a) arraigado sentimiento de pertenencia y cuentan con lazos y vínculos sociales sólidos como apoyo; (b) confianza en los demás como en sí mismos y aceptación de los aspectos positivos y negativos de su vida; (c) sentimiento de utilidad al interior del colectivo; (d) confianza en el futuro de la sociedad reconociendo su potencial de crecimiento y beneficios; y (e) concebir su vida y el mundo como algo lleno de sentido y objetivos (Keyes, 1998).

Nos enmarcamos en la Psicología Positiva la cual centra su trabajo en el estudio científico de las fortalezas y virtudes humanas, focalizado en la construcción de condiciones positivas por sobre el énfasis de remediar lo negativo (Seligman y Csikszentmihalyi, 2000). Su objetivo es que las personas experimenten más emociones positivas que negativas y alcancen un bienestar integral que potencie el florecer en múltiples áreas de la vida y de la experiencia humana. Sus aportes han permitido el surgimiento del comportamiento organizacional positivo, definido como el "estudio y aplicación de una orientación positiva hacia las fortalezas de los recursos 
humanos las cuales pueden ser medidas, desarrolladas y gestionadas efectivamente para mejorar el rendimiento en el lugar de trabajo" (Bakker y Oerlemans, 2011; Xanthopoulou, Bakker y llies, 2012).

En este contexto, el Bienestar Social en el trabajo constituye una variable clave del éxito organizacional por cuanto conlleva relaciones personales positivas y enriquecedoras, menor frecuencia y mejor resolución de conflictos e incrementa la innovación en el trabajo y participación cívica, lo que potencia el uso efectivo del tiempo laboral, aumento de la calidad del trabajo y compromiso con la organización (Czrew, 2019). Factores como compromiso laboral (Adler, 2012) y satisfacción laboral (Vacharkulksemsuk y Fredrickson, 2013) hacen referencia a juicios agradables, actitudes positivas o experiencias placenteras en el contexto del trabajo (Bakker y Oerlemans, 2011; Fisher, 2010). Asimismo, variables como eficacia, esperanza, optimismo y resiliencia conforman el capital psicológico con que cuentan las organizaciones (Luthans y Avolio, 2009). En las investigaciones realizadas es frecuente la inclusión de indicadores como: contacto social (Diener, 1994) y contacto interpersonal (Erikson, 1998) los cuales se complementan con los recursos sociales y resultan en relaciones positivas con los demás (Ryff y Keyes, 1995; Keyes, et al., 2002).

El Bienestar Social en el trabajo implica una interacción equilibrada entre demandas, entendidas como esfuerzo y costo físico, psicológico, emocional, cognitivo y conductual requerido al trabajador; y recursos como factores del contexto laboral que favorecen el hacer frente a las exigencias, facilitando el desempeño y crecimiento personal, y laboral (Garrosa y Carmona, 2011). Requiere movilizar factores organizacionales y sociales generadores de entornos positivos y potenciadores de desarrollo que permitan conciliar la vida laboral y personal (Garrosa y Carmona, 2011) lo que se constituye en factores protectores de los riesgos psicosociales en contexto laboral (Castro, 2018). Las personas con mayor apoyo social en el contexto del trabajo se sienten más queridas, más satisfechas y tienen menor riesgo de depresión (Marrero y Carballeira, 2010). Igualmente, una mayor colaboración, cooperación y resolución colaborativa de problemas, aumenta el nivel de confianza de los trabajadores, y eleva los niveles de productividad y calidad de vida (Unanue, Maercovich, Olguín y Weldt, 2013, p. 298). Dada la dificultad de encontrar instrumentos de bienestar social en contexto laboral, el objetivo de la presente investigación es adaptar y validar al contexto del trabajo la Escala de Bienestar Social "Social Well-Being Scale" creada por Keyes (1998) y adaptada al habla hispana por Blanco y Díaz (2005) cuyo objeto de análisis es la sociedad en la que se inserta el individuo.

\section{MÉTODO}

La investigación corresponde a un diseño de tipo instrumental transversal (Montero y León, 2002). La metodología se presenta en tres secciones: participantes, instrumento, procedimiento y análisis de datos.

\section{Participantes}

La muestra es de tipo no probabilística y se divide en dos submuestras. La primera de ellas, submuestra $\mathrm{A}$, se compone de 173 sujetos, $33.5 \% \%$ hombres $(n=58)$ y $66.5 \%$ mujeres $(n=115)$ con una edad media de 44.02 años (DT= 9.8). La submuestra B se compone de 159 sujetos, $33.3 \%$ mujeres $(n=53)$ y $66.7 \%$ hombres $(n=106)$, con edad media de 44.18 años (DT=6.2). Todos trabajadores activos de sectores productivos y de servicios, mayores de 18 años, con contrato laboral superior a tres meses.

\section{Instrumento}

Los 33 reactivos del instrumento original son de tipo diferencial semántico, hacen referencia a la sociedad en general y se integran en las siguientes dimensiones:1) Aceptación social: confianza, y actitud positiva hacia los otros, a quienes se les atribuye características positivas. Aceptación de los aspectos sociales positivos y negativos de nuestra propia vida; 2) Integración Social: calidad de las relaciones que el sujeto mantiene con la comunidad. Implica sentirse parte de la sociedad y tender lazos con diversos sistemas sociales; 3) Contribución social: sentimiento de utilidad, y contribución al bien común, y creencia que lo que uno aporta es valorado por otros; 4) Actualización social: creencia que la sociedad sabe dónde va, confianza en su potencial de desarrollo y en su capacidad para producir bienestar; y 5) Coherencia social: capacidad de otorgar sentido a lo que pasa en la sociedad y encontrar lógica en los acontecimientos que nos rodean (Keyes,1998).

\section{Procedimiento y análisis de datos}

En una primera instancia, con el apoyo de expertos en lingüística y psicología laboral se ejecuta el proceso de adaptación al contexto del trabajo de los 33 reactivos del instrumento de Bienestar Social desarrollado por Keyes (1998) y traducido por Blanco y Díaz (2005). La validez de contenido y constructo se confirma en base a jueces expertos, quienes realizan una evaluación y calificación del nivel de adecuación a las definiciones correspondientes y un análisis de la redacción sintáctica y semántica de los reactivos (Tabla 1). 
Tabla 1: Adaptación de Instrumento de Bienestar Social de Blanco y Díaz al contexto laboral

\begin{tabular}{|c|c|}
\hline \multicolumn{2}{|l|}{ Integración social } \\
\hline $\begin{array}{l}\text { 01. Siento que soy una parte importante de mi } \\
\text { comunidad. }\end{array}$ & 1. Siento que soy una parte importante de mi empresa. \\
\hline 02. Creo que la gente me valora como persona. & 2. Creo que en mi entorno laboral me valoran como persona. \\
\hline $\begin{array}{l}\text { 03. Si tengo algo que decir, creo que la mayoría de } \\
\text { la gente me escucharía. }\end{array}$ & $\begin{array}{l}\text { 3. Si tengo que decir algo, la mayoría de las personas de mi } \\
\text { entorno laboral me escucharía. }\end{array}$ \\
\hline 04. Me siento cercano a otra gente. & $\begin{array}{l}\text { 4. Me siento cercano a las personas que trabajan en esta } \\
\text { empresa. }\end{array}$ \\
\hline $\begin{array}{l}\text { 05. Si tuviera algo que decir, pienso que la gente } \\
\text { no se lo tomaría en serio. }\end{array}$ & $\begin{array}{l}\text { 5. Si tuviera algo que decir, las personas que trabajan en esta } \\
\text { empresa NO se lo tomarían en serio. }\end{array}$ \\
\hline 06. No me siento pertenecer a ningún grupo social. & 6. NO me siento perteneciente a esta empresa. \\
\hline $\begin{array}{l}07 \text {. La sociedad en la que vivo es una fuente de } \\
\text { bienestar. }\end{array}$ & 7. La empresa en la que trabajo es una fuente de bienestar. \\
\hline \multicolumn{2}{|l|}{ Aceptación social } \\
\hline 08. Creo que la gente no es de fiar. & 8. La gente de mi entorno laboral NO es de fiar. \\
\hline $\begin{array}{l}\text { 09. Creo que las personas sólo piensan en sí } \\
\text { mismas. }\end{array}$ & 9. En esta empresa las personas solo piensan en sí mismas. \\
\hline 10. Creo que no se debe confiar en la gente. & 10. NO se debe confiar en la gente de esta empresa. \\
\hline 11. Creo que la gente es egoísta. & 11. Creo que la gente de esta empresa es egoísta. \\
\hline $\begin{array}{l}\text { 12. Hoy en día, la gente es cada vez más } \\
\text { deshonesta. }\end{array}$ & $\begin{array}{l}\text { 12. Hoy en día, las personas de esta empresa son cada vez más } \\
\text { deshonestas. }\end{array}$ \\
\hline $\begin{array}{l}\text { 13. Las personas no se preocupan de los } \\
\text { problemas de otros. }\end{array}$ & $\begin{array}{l}\text { 13. Las personas de esta empresa NO se preocupan de los } \\
\text { problemas de otros. }\end{array}$ \\
\hline 14. Creo que las personas son amables. & 14. En esta empresa creo que las personas son amables. \\
\hline $\begin{array}{l}\text { 15. Las personas no esperan nada a cambio } \\
\text { cuando hacen un favor. }\end{array}$ & $\begin{array}{l}\text { 15. En esta empresa las personas NO esperan nada a cambio } \\
\text { cuando hacen un favor. }\end{array}$ \\
\hline \multicolumn{2}{|l|}{ Contribución social } \\
\hline 16. Creo que puedo aportar algo al mundo. & 16. Creo que puedo aportar algo en esta empresa. \\
\hline $\begin{array}{l}\text { 17. No tengo nada importante que ofrecer a la } \\
\text { sociedad. }\end{array}$ & 17. NO tengo nada importante que ofrecerle a esta empresa. \\
\hline $\begin{array}{l}\text { 18. Mis actividades diarias no aportan nada que } \\
\text { valga la pena a la sociedad. }\end{array}$ & $\begin{array}{l}\text { 18. Mis actividades diarias NO aportan nada que valga la pena a } \\
\text { esta empresa. }\end{array}$ \\
\hline $\begin{array}{l}\text { 19. No tengo ni el tiempo ni la energía para aportar } \\
\text { algo a la sociedad. }\end{array}$ & $\begin{array}{l}\text { 19. NO tengo ni el tiempo no la energía para aportar algo a esta } \\
\text { empresa. }\end{array}$ \\
\hline $\begin{array}{l}\text { 20. Pienso que lo que hago es importante para la } \\
\text { sociedad. }\end{array}$ & 20. Pienso que lo que hago es importante para esta empresa. \\
\hline $\begin{array}{l}\text { 21. Lo que hago tiene alguna influencia sobre otras } \\
\text { personas. }\end{array}$ & $\begin{array}{l}\text { 21. Lo que hago en esta empresa tiene alguna influencia sobre } \\
\text { otras personas. }\end{array}$ \\
\hline \multicolumn{2}{|l|}{ Actualización social } \\
\hline $\begin{array}{l}\text { 22. Para mí el progreso social es algo que no } \\
\text { existe. }\end{array}$ & $\begin{array}{l}\text { 22. Para mí el progreso social es algo que NO existe en esta } \\
\text { empresa. }\end{array}$ \\
\hline $\begin{array}{l}\text { 23. La sociedad no ofrece alicientes para gente } \\
\text { como yo. }\end{array}$ & 23. La empresa NO ofrece motivación para la gente como yo. \\
\hline $\begin{array}{l}\text { 24. Veo que la sociedad está en continuo } \\
\text { desarrollo. }\end{array}$ & 24. Veo que la empresa está en continuo desarrollo. \\
\hline $\begin{array}{l}\text { 25. No creo que instituciones como la justicia o el } \\
\text { gobierno mejoren mi vida. }\end{array}$ & $\begin{array}{l}\text { 25. NO creo que las normativas de esta empresa mejoren mi } \\
\text { vida. }\end{array}$ \\
\hline 26. La sociedad ya no progresa. & 26. Esta empresa ya NO progresa. \\
\hline $\begin{array}{l}\text { 27. El mundo es cada vez un lugar mejor para la } \\
\text { gente. }\end{array}$ & 27. Esta empresa es cada vez un lugar mejor para la gente. \\
\hline
\end{tabular}


Tabla 1 (continuación)

\begin{tabular}{|l|l|}
\hline Coherencia social \\
\hline 28. No entiendo lo que está pasando en el mundo. & $\begin{array}{l}\text { 28. NO entiendo lo que pasa en las distintas áreas de esta } \\
\text { empresa. }\end{array}$ \\
\hline 29. El mundo es demasiado complejo para mí. & 29. Esta empresa es demasiado compleja para mí. \\
\hline $\begin{array}{l}\text { 30. No merece la pena esforzarse en intentar } \\
\text { comprender el mundo en el que vivo. }\end{array}$ & $\begin{array}{l}\text { 30. NO vale la pena esforzarse en intentar comprender esta } \\
\text { empresa. }\end{array}$ \\
\hline $\begin{array}{l}\text { 31. Muchas culturas son tan extrañas que no puedo } \\
\text { comprenderlas. }\end{array}$ & $\begin{array}{l}\text { 31. Muchos elementos de esta empresa son tan extraños } \\
\text { que no puedo comprenderlos. }\end{array}$ \\
\hline $\begin{array}{l}\text { 32. Los científicos son los únicos que pueden entender } \\
\text { cómo funciona el mundo }\end{array}$ & $\begin{array}{l}\text { 32. Los administrativos son los únicos que pueden entender } \\
\text { cómo funciona esta empresa }\end{array}$ \\
\hline $\begin{array}{l}\text { 33. Me resulta fácil predecir lo que puede suceder en el } \\
\text { futuro. }\end{array}$ & $\begin{array}{l}\text { 33. Me resulta fácil predecir lo que puede suceder en el } \\
\text { futuro en esta empresa. }\end{array}$ \\
\hline
\end{tabular}

En base a un muestreo no probabilístico, psicólogos previamente capacitados aplican el instrumento adaptado al contexto laboral, entregando las instrucciones de manera oral. La aplicación es realizada de manera grupal dentro de la jornada laboral de los participantes, con una duración de 15 minutos aproximadamente. Cada actor social responde vía lápiz y papel de manera autónoma. Todos los participantes accedieron a ser parte de la investigación de forma voluntaria, lo cual fue corroborado mediante consentimientos informados individuales (i.e. participación voluntaria, consecuencias de la aplicación, garantías de resguardo de la identidad, acceso y fines de la información). Por su parte, cada organización firmó los consentimientos correspondientes a nivel institucional.

La versión adaptada con los 33 ítems se aplica a la submuestra A. Luego se ejecuta un análisis de ítems y una depuración mediante análisis factorial exploratorio con el programa IBM SPSS Statistics V.22, mediante mínimos cuadrados generalizados. La validez de constructo se comprueba mediante Análisis de Componentes Principales y Rotación Normalización Varimax, que se suma a la validez de contenido. Mediante el coeficiente Alfa de Cronbach se determinó la consistencia interna estableciendo su fiabilidad. Posteriormente se aplica la versión depurada (17 ítems) a la submuestra B, y se establecen las estimaciones de fiabilidad mediante el coeficiente omega y el análisis factorial confirmatorio con el método de estimación mínimos cuadrados ponderados multivariantes a través del programa MPLUS v. 7.4.

En la interpretación de los datos se consideran los siguientes parámetros: coeficiente Alfa aquellos valores iguales o superiores a 0.75 corresponden a confiabilidad aceptable y valores igual o superior a 0.90 equivalen a confiabilidad elevada. Por otra parte, para Root Mean Square Error of Approximation (RMSEA) los valores menores a 0.05 indican ajuste adecuado y valores entre 0.05 y 0.08 ajuste razonable (Browne y Cudeck, 1993). Para Comparative Fix Index (CFI) e Index Tucker-Lewis (TLI), se considera que los valores iguales o mayores a 0,95 presentan un buen ajuste (Ruiz, Pardo y San Martín, 2010). Finalmente, especto al coeficiente omega valores entre 0.70 y 0.90 constituyen un valor aceptable (Oviedo y Campus-Arias, 2005).

\section{RESULTADOS}

Los resultados que se presentarán a continuación se dividirán de acuerdo a los dos análisis realizados: análisis factorial exploratorio y análisis factorial confirmatorio.

\section{Análisis Factorial Exploratorio}

Se realiza un examen inicial que arroja datos adecuados para las técnicas de análisis factorial exploratorio $(\mathrm{KMO}=0.803)$. La validez de constructo se comprueba mediante Análisis de Componentes Principales y Rotación Normalización Varimax, consolidándose 17 ítems organizados en tres dimensiones: 1) Interacción Social en el trabajo con 8 ítems, $(\alpha=0.95)$; 2) Pertenencia Social en el trabajo con 5 ítems $(\alpha=0.787)$; y 3) Comprensión Social en el trabajo con 4 ítems $(\alpha=0.797)$. El valor de Alfa de Cronbach de la escala total compuesta por 17 ítems puntúa 0.942 otorgando un índice adecuado de consistencia interna. Lo que, sumado a la validez de contenido, confirma características psicométricas adecuadas para su uso. En la Tabla 2 se presenta el Análisis de Componentes Principales. El total de varianza explicada por los 3 componentes principales de la Escala de Bienestar Social en el Trabajo es de 68.37\%.

Debido a la reorganización en tres componentes se redefine el constructo de bienestar social en el trabajo entendiéndose como la valoración positiva que los trabajadores hacen de sus circunstancias y funcionamiento social dentro del contexto laboral y se compone por: 1) Interacción Social en el Trabajo: valoración positiva 
de las cualidades de los compañeros de trabajo y de la organización como sistema social, en cuanto a la calidad de los vínculos y de las relaciones interpersonales que se establecen; 2) Pertenencia Social en el Trabajo: valoración positiva del grado en que los trabajadores se sienten involucrados con la organización, manteniendo un sentimiento de utilidad hacia ésta, favoreciendo su fidelidad a la misma; 3) Comprensión Social en el Trabajo: valoración positiva del nivel de entendimiento que tiene el trabajador acerca del funcionamiento social y administrativo de la organización, permitiéndole entender lo que acontece a nivel de vínculos sociales en el entorno laboral.

Tabla 2: Análisis de Componentes Principales de la Escala de Bienestar Social en el Trabajo

\begin{tabular}{|c|c|c|c|}
\hline \multirow[b]{2}{*}{ Ítem } & \multicolumn{3}{|l|}{ Factor* $^{*}$} \\
\hline & $\begin{array}{l}1 \text { (Interacción } \\
\text { Social en el } \\
\text { Trabajo) }\end{array}$ & $\begin{array}{l}2 \text { (Pertenencia } \\
\text { Social en el } \\
\text { Trabajo) }\end{array}$ & $\begin{array}{l}3 \text { (Comprensión } \\
\text { Social en el } \\
\text { Trabajo) }\end{array}$ \\
\hline 1. Creo que la gente de esta empresa es egoísta. & 0.862 & & \\
\hline 2. NO se debe confiar en la gente de esta empresa. & 0.834 & & \\
\hline $\begin{array}{l}\text { 3. Las personas de esta empresa NO se preocupan } \\
\text { de los problemas de otros. }\end{array}$ & 0.762 & & \\
\hline $\begin{array}{l}\text { 4. Hoy en día, las personas de esta empresa son } \\
\text { cada vez más deshonestas. }\end{array}$ & 0.757 & & \\
\hline 5. La gente de mi entorno laboral NO es de fiar. & 0.728 & & \\
\hline $\begin{array}{l}\text { 6. En esta empresa las personas solo piensan en sí } \\
\text { mismas. }\end{array}$ & 0.720 & & \\
\hline $\begin{array}{l}\text { 7. NO vale la pena esforzarse en intentar } \\
\text { comprender esta empresa. }\end{array}$ & 0.667 & & \\
\hline 8. Esta empresa ya NO progresa. & 0.586 & & \\
\hline $\begin{array}{l}\text { 9. NO tengo nada importante que ofrecerle a esta } \\
\text { empresa. }\end{array}$ & & 0.740 & \\
\hline $\begin{array}{l}\text { 10. NO tengo ni el tiempo no la energía para aportar } \\
\text { algo a esta empresa. }\end{array}$ & & 0.703 & \\
\hline 11. NO me siento perteneciente a esta empresa. & & 0.668 & \\
\hline $\begin{array}{l}\text { 12. La empresa NO ofrece motivación para la gente } \\
\text { como yo. }\end{array}$ & & 0.642 & \\
\hline $\begin{array}{l}\text { 13. NO creo que las normativas de esta empresa } \\
\text { mejoren mi vida. }\end{array}$ & & 0.607 & \\
\hline $\begin{array}{l}\text { 14. NO entiendo lo que pasa en las distintas áreas } \\
\text { de esta empresa. }\end{array}$ & & & 0.751 \\
\hline $\begin{array}{l}\text { 15. Los administrativos son los únicos que pueden } \\
\text { entender cómo funciona esta empresa. }\end{array}$ & & & 0.749 \\
\hline $\begin{array}{l}\text { 16. Muchos elementos de esta empresa son tan } \\
\text { extraños que no puedo comprender los }\end{array}$ & & & 0.686 \\
\hline 17. Esta empresa es demasiado compleja para mí. & & & 0.565 \\
\hline Porcentaje de varianza explicada & $30.26 \%$ & $20.92 \%$ & $17.19 \%$ \\
\hline
\end{tabular}

\section{Análisis Factorial Confirmatorio}

El modelo obtenido se pone a prueba con la submuestra B con el fin de determinar su estabilidad factorial mediante un análisis factorial confirmatorio (AFC). El AFC realizado a la Escala de 17 ítems, arroja valores que no se ajustan adecuadamente por lo que se extraen los siguientes reactivos, que presentan cargas cruzadas: Ítem 8 ("No tengo nada importante que ofrecerle a esta empresa"), 12 ("Está empresa ya no progresa"), 14 ("Esta empresa es demasiado compleja para mí") y 15 ("No vale la pena esforzarse en intentar comprender esta empresa"), obteniendo una Escala de 13 ítems. Esta versión final de la Escala de Bienestar Social en el Trabajo con 13 reactivos presenta valores CFI y TLI con buen ajuste. EI RMSEA arroja valores con mejor nivel de ajuste en comparación a la escala de 17 ítems, optimizando la estabilidad factorial (Tabla 3). 
Tabla 3: Indicadores de Ajuste Escala de Bienestar Social en el Trabajo

\begin{tabular}{|l|c|c|}
\hline Índice de Ajuste & Modelo 17 ítems & Modelo 13 ítems \\
\hline Comparative fix index (CFI) & 0.943 & 0.984 \\
\hline Index Tucker-Lewis (TLI) & 0.935 & 0.980 \\
\hline Root Mean Square Error of Approximation (RMSEA) & 0.137 & 0.091 \\
\hline
\end{tabular}

En la Figura 1 se observa la representación gráfica de la versión final de la Escala de Bienestar Social en el Trabajo. Se confirman las 3 dimensiones establecidas en el AFE: Pertenencia Social en el Trabajo con un total de 4 ítems, Interacción Social en el Trabajo compuesta por 6 ítems y Comprensión Social en el Trabajo integrada por 3 ítems. La consistencia interna para cada una de las tres dimensiones se obtuvo con la totalidad de la submuestra B determinándose los Alfa de Cronbach y Omegas que se presentan en la Tabla 4.

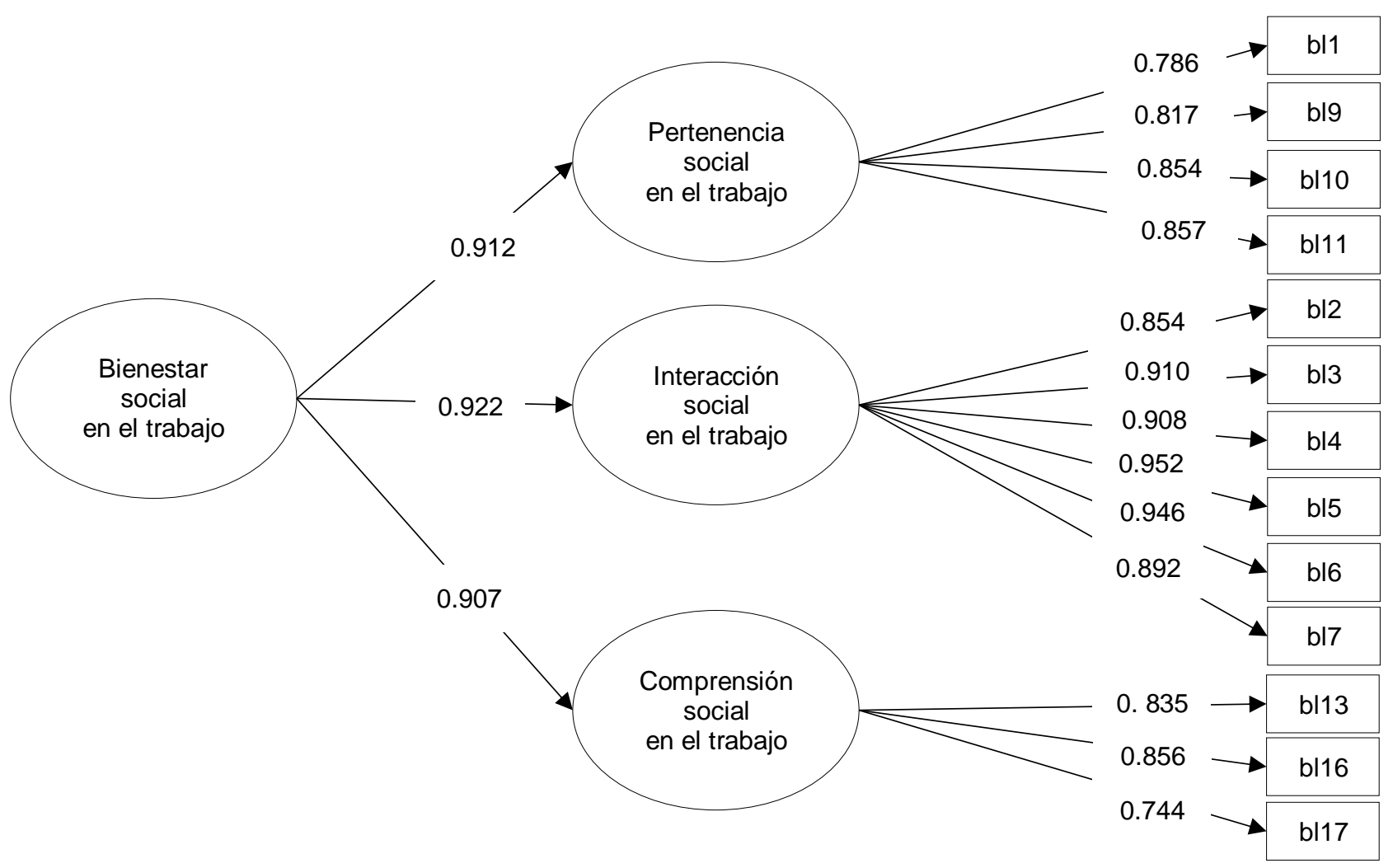

Fig. 1 Representación del Modelo de Bienestar Social en el Trabajo y sus estimaciones de saturaciones factoriales

Tabla 4: Coeficientes de Fiabilidad: Alfa de Cronbach y Omega

\begin{tabular}{|l|l|l|l|}
\cline { 2 - 4 } \multicolumn{2}{c|}{} & \multicolumn{2}{l|}{ Dimensión } \\
\hline Estadístico & $\begin{array}{l}\text { Pertenencia social } \\
\text { en el trabajo }\end{array}$ & $\begin{array}{l}\text { Interacción social } \\
\text { en el trabajo }\end{array}$ & $\begin{array}{l}\text { Comprensión social } \\
\text { en el trabajo }\end{array}$ \\
\hline Alfa de Cronbach & 0.813 & 0.937 & 0.780 \\
\hline Omega & 0.898 & 0.967 & 0.854 \\
\hline
\end{tabular}

La escala total arroja un Alfa de Cronbach de 0.938 certificando buena consistencia interna, al igual que cada una de sus dimensiones. Los ítems se puntúan de 4 a 1, en donde 1 corresponde a totalmente de acuerdo, 2 de acuerdo, 3 en desacuerdo y 4 totalmente en desacuerdo. Para establecer las puntuaciones de la escala se utilizó puntuaciones típicas o estándar lineales directos. Estas calificaciones señalan la distancia, en términos de desviaciones típicas, en que se encuentra un puntaje original con respecto a la media de su distribución de puntajes originales, en el caso de la submuestra B corresponde a la media $(X=42.99)$ y la desviación estándar $(\sigma=7.43)$. La suma por dimensiones permite obtener un registro ordinal que va desde un 
mínimo de 13 puntos en el extremo inferior hasta un puntaje máximo de 52 puntos en el extremo superior. Los trabajadores de la submuestra B que alcanzan puntajes inferiores a 35.56 revelan Bajo Bienestar Social en el Trabajo, puntajes superiores a 50.42 puntos equivalen a un nivel Alto, mientras que aquellas personas que fluctúan entre 50.42 y 35.56 corresponden a un rango Medio.

\section{DISCUSIÓN}

La escala de Bienestar Social en el Trabajo se revela como un instrumento que permite levantar datos válidos y confiables que orienten procesos de intervención y desarrollo organizacional (Tabla 5). El Bienestar Social en el Trabajo apunta a factores organizacionales, laborales y sociales que generan posibilidades de desarrollo y entornos amigables donde el trabajador puede conciliar en mayor medida su vida (Garrosa y Carmona, 2011). Al igual que lo planteado por Martínez, Ivanovic-Zuvic y Unanue (2013), aquellos sistemas sociales que generan mayor Bienestar Social en el Trabajo promoverán una mayor disposición a la cooperación y resolución colaborativa de conflictos, lo que potencia la confianza de los trabajadores, productividad organizacional y calidad de vida de las personas.

Tabla 5: Escala de Bienestar Social en el Trabajo

\begin{tabular}{|c|c|c|c|c|}
\hline \multicolumn{5}{|c|}{ EBS.CL } \\
\hline \multicolumn{5}{|c|}{$\begin{array}{l}\text { Instrucciones: Señale con una " } X \text { " su nivel de acuerdo con cada una de las afirmaciones que a continuación se hacen } \\
\text { referente a la empresa en que trabaja. Debe contestar frente a todas las afirmaciones. No dejar ninguna en blanco. }\end{array}$} \\
\hline & \multicolumn{4}{|c|}{ Escala de Elección } \\
\hline Ítems & $\begin{array}{l}\text { Totalmente en } \\
\text { Desacuerdo }\end{array}$ & $\begin{array}{l}\text { En } \\
\text { Desacuerdo }\end{array}$ & $\begin{array}{l}\text { De } \\
\text { Acuerdo }\end{array}$ & $\begin{array}{l}\text { Totalmente de } \\
\text { Acuerdo }\end{array}$ \\
\hline 1. NO me siento perteneciente a esta empresa. & & & & \\
\hline 2. La gente de mi entorno laboral NO es de fiar. & & & & \\
\hline $\begin{array}{l}\text { 3. En esta empresa las personas solo piensan en } \mathrm{s} \\
\text { mismas. }\end{array}$ & & & & \\
\hline 4. NO se debe confiar en la gente de esta empresa & & & & \\
\hline 5. Creo que la gente de esta empresa es egoísta. & & & & \\
\hline $\begin{array}{l}\text { 6. Hoy en día, las personas de esta empresa son } \\
\text { cada vez más deshonestas. }\end{array}$ & & & & \\
\hline $\begin{array}{l}\text { 7. Las personas de esta empresa NO se preocupar } \\
\text { de los problemas de otros. }\end{array}$ & & & & \\
\hline $\begin{array}{l}\text { 8. NO tengo ni el tiempo ni la energía para aportar } \\
\text { algo a esta empresa. }\end{array}$ & & & & \\
\hline $\begin{array}{l}\text { 9. La empresa NO ofrece motivación para la gente } \\
\text { como yo. }\end{array}$ & & & & \\
\hline $\begin{array}{l}\text { 10. NO creo que las normativas de esta empresa } \\
\text { mejoren mi vida. }\end{array}$ & & & & \\
\hline $\begin{array}{l}\text { 11. NO entiendo lo que pasa en las distintas áreas } \\
\text { de esta empresa. }\end{array}$ & & & & \\
\hline $\begin{array}{l}\text { 12. Muchos elementos de esta empresa no puedo } \\
\text { comprenderlos. }\end{array}$ & & & & \\
\hline $\begin{array}{l}\text { 13. Los administrativos son los únicos que pueden } \\
\text { entender cómo funciona esta empresa. }\end{array}$ & & & & \\
\hline
\end{tabular}

Las dimensiones de Interacción Social, Pertenencia Social y Comprensión Social en el Trabajo confirman lo planteado por el MIT referente a que el bienestar de un sistema social aumenta cuando los participantes poseen y desarrollan sus habilidades colectivas y sensibilidad social, co-construyen confianzas y se brindan la posibilidad de que puedan compartir en tiempo real y en paralelo, sin barreras ni restricciones, sus ideas y conocimientos, beneficiándose las empresas al aumentar su producción de valor, innovación, generando equipos más productivos que reportan mejor calidad de vida en el trabajo (Muzard, 2012). 


\section{CONCLUSIONES}

Del proceso de adaptación y validación a contextos laborales transversales chilenos de la escala de bienestar social propuesta por Keyes (1998) y adaptada al habla hispana por Blanco y Díaz (2005) se puede concluir lo siguiente: 1.- La escala de bienestar social en el trabajo (EBS:CL), posee características psicométricas adecuadas para su uso constituyendo un aporte al diagnóstico e intervención de sistemas laborales y a la Psicología del Trabajo y las Organizaciones; 2.- Este instrumento constituye un aporte al desafío de construir un modelo de desarrollo que atienda las demandas de los trabajadores a partir de la pregunta por el vínculo social y las maneras de construir capacidades de acción colectiva.

\section{REFERENCIAS}

Adler, S., Work Engagement: A Handbook of Essential Theory and Research, Bakker, A. B., y Leiter, M. P. (Ed.), Personnel Psychology: The Study of People at Work, 204-207, Psychology Press, Nueva York, Estados Unidos (2012)

Arévalo-Avecillas, D., Game, C., Padilla-Lozano, C., y Wong, N. Predictores de la Calidad de Vida Subjetiva en Adultos Mayores de Zonas Urbanas y Rurales de la Provincia del Guayas, Ecuador, doi: 10.4067/S0718-07642019000500271, Información Tecnológica, 30(5), 271-282 (2019)

Bakker, A., B., y Oerlemans W. G. M., Subjective Well-being in Organizations, Spreitzer, G. M. y Cameron, K. S., (Ed.), The oxford Handbook of Positive Organizational Scholarship, 178-189, Oxford University Press, Nueva York, Estados Unidos (2011)

Blanco, A., y Díaz, D., El Bienestar Social: Su Concepto y Medición, ISSN: 1886-144X, Psicothema, 17(4), $582-584$ (2005)

Basaure, M., Archivos de Frontera: El Gobierno de las Emociones en Argentina y Chile del Presente, doi: http://dx.doi.org/10.4067/S0718-65682013000100026, Polis, 12(34), 543-548 (2013)

Browne, M. W. y Cudeck, R., Alternative Ways of Assessing Model Fit, Bollen, K. y Long J. (Ed.), Testing Structural Equation Models, 136-162, Sage, California, Estados Unidos (1993)

Castellanos, R., Comprendiendo la Relación entre Bienestar Subjetivo, Cohesión y Relaciones Sociales: Una Aproximación al Caso México y sus Entidades Federativas, ISSN: 1696-1277, Revista Circunstancia, 11(3), 2-19 (2013)

Castro, N. P., Riesgos Psicosociales y Salud Laboral en Centros de Salud, doi: https://dx.doi.org/10.4067/S071824492018000300155, Ciencia y trabajo, 20(63), 155-159 (2018)

Czerw, A., Diagnosing Well-Being in Work Context-Eudemonic Well-Being in the Workplace Questionnaire, doi: 10.1007/s12144-017-9614-8, Current Psychology, 38, 331-346 (2019)

Diener, E. F., Assessing Subjective Well-Being: Progress and Opportunities. Social Indicators, doi: https://doi.org/10.1007/BF01207052, Research, 31(2), 103-157 (1994)

Erikson, R., Descripciones de la Desigualdad: El Enfoque Sueco de las Investigaciones de Bienestar Sen, A. K. y Nussbaum, M. C., La calidad de vida, 101-120, Fondo de cultura económica, México (1998)

Fischer, C. D., Happiness at Work, doi: https://doi.org/10.1111/j.1468-2370.2009.00270.x, International Journal of Management Reviews, 12(4), 384-412 (2010)

Garrosa, E., y Carmona, I., Salud Laboral y Bienestar. Incorporación de Modelos Positivos a la Comprensión y Prevención de los Riesgos Psicosociales del Trabajo, doi: 10.4321/S0465-546X2011000500014, Medicina y Seguridad del Trabajo, 57(1), 224-238 (2011)

Ivankina, L., y Latygovskaya, T., Modern Social Welfare in the light of the sustainability model, doi: 10.1016/j.sbspro.2014.12.493, Procedia Social Behavioral Sciences, 166, 111-115 (2015)

Keyes, C. L. M., Shmotkin, D., y Ryff, C. D., Optimizing Well-being: The Empirical Encounter of Two Traditions, doi: 10.1037/0022-3514.82.6.1007, Journal of Personality and Social Psychology, 82(6), 1007-1022 (2002)

Keyes, C. L. M., Social Well-Being, doi: 10.2307/2787065, Social Psychology Quarterly, 61(2), 121-140 (1998)

Luthans F., y Avolio, B. J., The "Point" of Positive Organizational Behavior, doi: 10.1002/job.589, Journal of Organizational Behavior, 30,291-307 (2009)

Marrero R. J., y Carballeira, M., El Papel del Optimismo y del Apoyo Social en el Bienestar Subjetivo, ISSN: 0185-3325, Salud Mental, 33(1), 39-46 (2010)

Martínez, D., Ivanovic-Zuvic, F., y Unanue, W., Introducción, Martínez, D., Ivanovic-Zuvic, F., y Unanue, W. La felicidad: Evidencias y experiencias para cambiar nuestro mundo, 23-35, Sonepsyn, Santiago, Chile (2013)

Martínez, D., y Zamora, L. Mirada I, Martínez, D., Ivanovic-Zuvic, F., y Unanue, W. La felicidad: Evidencias y experiencias para cambiar nuestro mundo, 119-125, Sonepsyn, Santiago, Chile (2013)

Montero, I., y León, O., Clasificación y Descripción de las Metodologías de Investigación en Psicología, ISSN: 2174-0852, Revista Internacional de Psicología Clínica y de la Salud, 2(3), 503-508 (2002)

Muzard, J. Trabajo colaborativo en tiempo real en equipo sin barreras mediatizado por una interface virtual compartida online, $6^{\circ}$ Seminario de Acercamiento Tecnológico en el Área Capital Humano en Calama, Chile (2012) 
Oviedo, H. C., y Campo-Arias, A., Aproximación al Uso del Coeficiente Alfa de Cronbach, ISSN:0034-7450, Revista Colombiana de Psiquiatría, 34, (4), 572-580 (2005)

Programas de las Naciones Unidas para el Desarrollo, Informe Sobre Desarrollo Humano 2012: Trabajo al Servicio del Desarrollo Humano, Programas de las Naciones Unidas para el Desarrollo (2012)

Ruiz, M. A., Pardo, A., y San Martín, R., Modelos de ecuaciones estructurales, ISSN: 0214 - 7823, Papeles del Psicólogo, 31(1), 34-45 (2010)

Ryff, C. D., y Keyes, C. L. M., The Structure of Psychological Well-being Revisited, doi: 10.1037/0022-3514.69.4.719 Journal of Personality and Social Psychology, 69(4), 719-727 (1995)

Seligman, M. E. P., y Csikszentmihalyi, M., Positive Psychology, doi: 10.1037/0003-066X.55.1.5, American Psychologist, 55(1), 5-14 (2000)

Unanue, W., Maercovich, P., Olguín, E., y Weldt, C., Felicidad, Bienestar y Desempeño Organizacional, Martínez, D., Ivanovic-Zuvic, F., y Unanue, W. La felicidad: Evidencias y experiencias para cambiar nuestro mundo, 297-319, Sonepsyn, Santiago, Chile (2013)

Vacharkulksemsuk, T., y Fredrickson, B., Looking back and glimpsing forward: The broaden and build theory of positive emotions as applied to organizations, doi: 10.1108/S2046410X0000001005, Advances in Positive Organizational Psychology, 1, 45-60 (2013)

Xanthopoulou, D., Bakker, A. B., y llies, R., Everyday Working Life: Explaining Within-Person Fluctuations in Employee Well-Being, doi: 10.1177/0018726712451283, Human Relations, 65(9), 1051-1069 (2012) 